\title{
Highly Available XenApp Cloud
}

\author{
Isha $^{1}$ and Chander Kant ${ }^{2}$ \\ ${ }^{1,2}$ Deptt. of Computer Science \& Applications, Kurukshetra University, \\ Kurukshetra-136119 \\ ${ }^{1}$ reply2ishainegmail.com \\ ${ }^{2}$ ckvermalrediffmail.com
}

\begin{abstract}
In Cloud computing, application and desktop delivery are the two emerging technologies that has reduced application and desktop computing costs and provided greater IT and user flexibility compared to traditional application and desktop management models. Among the various SaaS technologies, XenApp that allow numerous end users to connect to their corporate applications from any device. XenApp enables organizations to improve application management by centralizing applications in the datacenter to reduce costs, controlling and encrypting access to data and applications to improve security and delivering applications instantly to users anywhere by remotely accessing or streaming. As per old architecture we were using the oracle or MS-SQL in the backend of XenApp on Windows Server itself. But as we know for this we have to pay for database especially because we are not using it for any other purpose. And as we know windows is GUI based so running database over it consumes much more resources. So, it can lead to single point of failure. For this, this paper proposes a scheme for removing this problem by using the HA failover cluster based SQL server (mysql or Oracle) which will run over Linux box having concept of VIP (Virtual IP).
\end{abstract}

\section{KEYWORDS}

Clustering, High-availability, Virtual IP

\section{INTRODUCTION}

Cloud can be viewed as a new frontier of the IT services based on its abilities to deliver cost effective, enterprise class virtual desktop and application solutions as provided by Citrix XenDesktop. Increased challenges and changing business needs has presented a challenge for IT staff to satisfy their users with services that incorporate the fundamentals of access at anytime from anywhere, with any device and ensuring secure access to business-critical applications and information. XenApp gives the ability to centrally manage heterogeneous applications and deliver Software as a Service (SaaS) to any workforce.

In classical computer architecture, applications are installed, managed and upgraded individually on every desktop. By the introduction of virtualization, an application need not to be managed individually as is done in centralized manner. With the increase in usage of variety of applications by an increased no. of users, has led to increase in no. of physical servers, need of high availability, load balancing, management of large amount of data and its reliability. Citrix XenApp server architecture has been succeeded to achieve this up to much extent. But there also complexity and cost are hinders the growth of organizations implementing this architecture. Citrix XenApp server hosts applications which are installed on servers in the data center and then accessed via any client device. And the shared desktop capability in XenApp gives IT the ability to provide a standardized, server OS desktop with installed applications to users, providing a consistent workspace from anywhere on demand[1].Earlier XenApp was used to be installed on 
physical servers, then to accomplish present scenario needs like need to scale quickly to handle rapid growth employee numbers, to reproduce issues without adding new hardware and to reduce physical server counts etc. , it is installed now on Virtual machines.[2] Its centralized application management and session virtualization management makes it most secure technique for delivering and streaming applications to any client device as data remains in the data center whereas the input which are in form of keystrokes and mouse clicks and the output screenshots are transit through the network at fast speed in an encrypted manner. At all XenApp provides the ability for application deployment, remote office connectivity, workforce mobility, business continuity [3].

\section{THE STANDARD XENAPP ARCHITECTURE}

XenApp is an enhanced form of Windows terminal services and an application hosting technology that utilizes Citrix Systems' proprietary presentation layer protocol or thin client protocol called Independent Computing Architecture (ICA)as shown in fig.1[4].

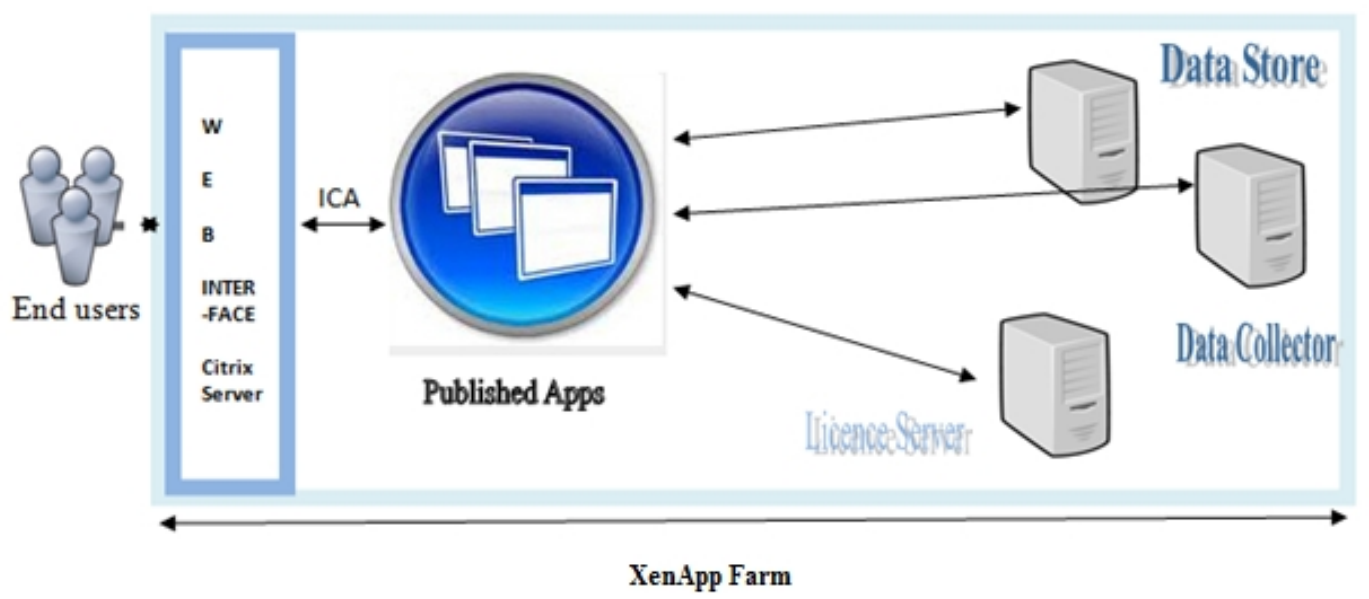

Figure 1: XenApp Server Architecture

The protocol lays down a specification for passing data between server and clients. A group of servers running XenApp shares common data store, known to be as Server farm. Servers are grouped into Zones which share common data store where applications can be load balanced across servers in farm. There can be multiple farms, where farms do not share data store and load balancing is done in individual farms [5]. Each Server farm consists of Farm servers, popularly known as Citrix XenApp servers, IMA database, the web interface and the access management console.

A The core components of citrix server include Multiwin, ICA and IMA. Multiwin is a kernel module that enables multiple concurrent users' login and access applications in separate secure session on a single server. This XenApp presentation server is hosted over Microsoft's Windows server which enables it to create multiple sessions on single instance of the operating system and by the help of its terminal services that makes it possible to connect to a session from any location and many devices. ICA works to separate the application's logic from the user interface then transports it to the client over standard network protocols- TCP/IP, PPP, etc. At the client side, users see and work with the application's interface, but the whole application logic is executed on the server [6]. It can be used with slowest as $14.4 \mathrm{kbps}$ to fastest up to Gbps speedy connections. This protocol allows up to 32-bit applications run over multi platform clients and establishes 
communication through 32 virtual channels. It is the only protocol used for client to server session secure communication run over basic network layer protocols. At the client side, a file with extension ".ica" is send by servers for application delivery services during a session. IMA known as Independent management architecture framework designed for server to server communication over data layer.

B The non-core components included in architecture are License Server, Web Interface, Data Collector, data store. [12] License Server is associated with license management console. It stores the license file and vital information about the product edition and the number of concurrent users viable and the expiry dates etc. The license management console manages license server and maintains license files for XenApp servers. The licenses are not associated with particular clients but a particular client-server combination is viable for reservation of license. It is returned to license pool after a user logoff its session for other users' usage purpose. Web Interface provides users with access to content via internet explorer to XenApp applications and XenDesktop virtual desktops through a graphical presentation. The Web Interface interacts with a XenApp Server to deliver its resources to the client device by installing online plug-in and other necessary configurations to the client device [7]. Web interface also performs user authentication checks using RSA Secure ID and by other means. Data Store is the most important database that holds configurations data, persistent information about farm servers, farm management security, published applications and server administrators and printers. Each zone contains a data collector to keep dynamic data for servers in Zone. These data collectors also act as communication gateways between Zones [8]. Data store used can be MySq1 Server Database over XenApp server.

C Application Delivery Process: The following figure shows how the XenApp architecture provides the ability to configure, manage, and enable application access from one centralized location, reducing the cost of provisioning offices individually and facilitating secured access from anywhere by any device.[9]

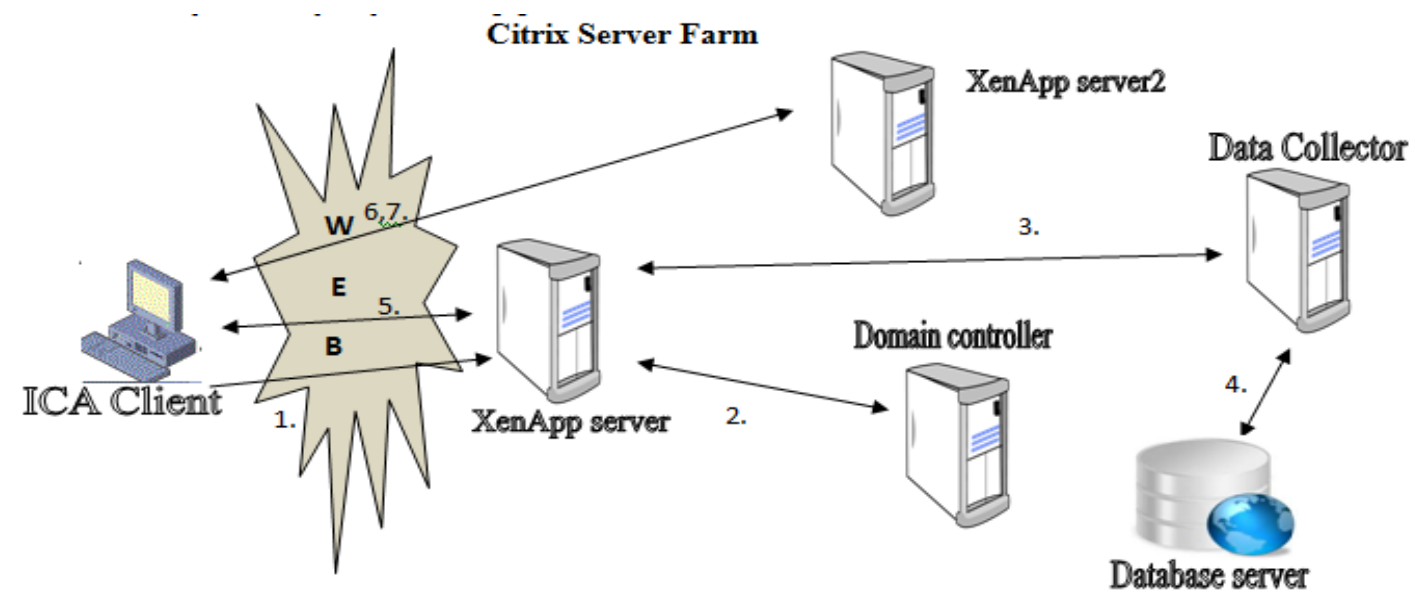

Figure 2: Steps in Application delivery process

This Application delivery process starts with the request from ICA client to XENAPP server for application access through Web interface by an ICA connection in $1^{\text {st }}$ step. Then the user is authenticated and verified if it is authorized to access the application and any concurrent user license is free for use or not by license server and domain controller[10] [11]. In the $3^{\text {rd }}$ step, request is forwarded to data controller to check which server has the application requested and which server can now serve the application. Then the data store is checked for configuration data 
and access management console is accessed to deliver the application according to user profile and its privileges. Then the client is redirected to idle server or least loaded server. Then the client requests for IMA service to establish an application delivery session in step 6.Now session is established over ICA connection and all inputs and outputs are communicated over ICA virtual channel. It also gives option for streaming of application at client device to work in offline manner[12].

D Database servers: Each farm requires a dedicated database. XenApp mostly uses Microsoft Sql Server and Oracle for managing Databases. However, multiple databases can be running on a single server running Microsoft SQL Server. The farm doesn't use a database that is shared with any other client/server applications. Here distributed databases are used. When there are too many requests for access the data store, it creates bottleneck. Microsoft SQL Server uses replication to create the distributed database environment. XenApp requires data coherency across multiple databases [13]. Therefore, a two-phase commit algorithm is required for storing data in the database. In existing architecture database server is installed on same machine on which XenApp is running as



Figure 3: A XenApp farm shows running both servers over same machine shown in figure 3.

There are two protocols used to connect to a database are TCP/IP sockets and named pipes. TCP/IP sockets do not depend on Windows authentication to establish a connection, but it provides username and password for the authentication to the database after the connection is established. Windows authentication reduces the possibility of an error occurring when the server hosting SQL Server and the XenApp server do not have the correct domain or Active Directory trust relationship. Named pipes is an authenticated communication protocol, so any time for an attempt to open a connection to the SQL Server database using this protocol, the Windows authentication process occurs[14]. Most database maintenance requires running the dsmaint and $d s c h e c k$ server utilities on XenApp farm servers. If the data store fails, each farm server can access the data in its Local Host Cache indefinitely, provided it can contact the license server. However, it cannot make any modifications to the farm. A back up copy is always maintained of data store, else user need to create all the farm policies, account, settings and data in the data store again and again. Migration is also needed sometimes for load balancing, it is done by the help of $d$ smaint migrate utility. There are also other utilities for checking the integrity 
of the server farm data store, to generate log reports to view information about server farms, processes, ICA sessions, users [15].

\section{PROPOSED XENAPP CLOUD ARCHITECTURE}

As per old architecture we were using the oracle or MS-SQL in the backend of XenApp on Windows Server itself. But as we know for this an organization has to pay for database especially because we are not using it for any other purpose. And as we know windows is GUI based so running database over it consumes much more resources. So, it can lead to single point of failure. Generally lead to service hang-ups on application publishing \& other changes. So, we are purposing to use cluster based sql servers in backend of XenApp. Which are typically based on NLB (Network Load Balancers) on windows. But again resource utilization \& license become the most important concern. For this we can remove this problem by using the HA failover cluster based SQL server(mysql or Oracle) which will run over Linux box having concept of VIP(Virtual Internet Protocol address).Since the Microsoft clustering is not reliable ,so we build cluster over Linux. That will be connected to windows xenapp server via "Ora Client" Application. A failover cluster is a combination of one or more physical disks in a cluster group that are participating group nodes of a cluster. The network is configured as a failover clustered instance that hosts an instance of SQL Server. A SQL Server failover clustered instance appears on the network as if it were a single computer, but has functionality that provides failover from one node to another if one node becomes unavailable. Before the cluster has time to fail over, the loss of the principal server is detected by the mirror server, with the help of the witness. The mirror server rolls forward its database and brings it online as the new principal database as quickly as possible. The principle server is the one serving the end user. And mirror server is replication of principal server and witness server keep monitoring the principal server so as to handover the access to mirror server. Now the problem is which IP should be provided to XenApp to allow the access to database. This IP will be called as Virtual IP since it is not the real IP of the database. This is done by the help of virtual IP. This "ora client" is an interface for accessing oracle database.

This mysql or Oracle can be used for other purposes too like storing other data. This will remove the exception of single point of failure as this will use SNMP or heartbeat. It will continuously monitor the service. We can typically setup any number of nodes typically 2 nodes for a small setup \& 5-7 for medium based, 10-15 for Enterprise based. But ORACLE charges according to number of nodes. So either use mysql or use ORACLE for other purposes too which is running on Linux. So, for this setup we have to install SQL on Linux server nodes, configure HA failover on those nodes, and install XenApp \& Ora Client on windows Servers. We have to specify VIP (virtual IP) of SQL in Ora client so that it will create an environment like sql is directly installed on the Microsoft Server. In this way backend database \& XenApp performance can be increased. This performance factor really does matter on production Servers (Generally where the enterprise can't afford downtime like on e-commerce servers).Following figure shows the new proposed architecture that implements HA failover clustering over Linux servers. 


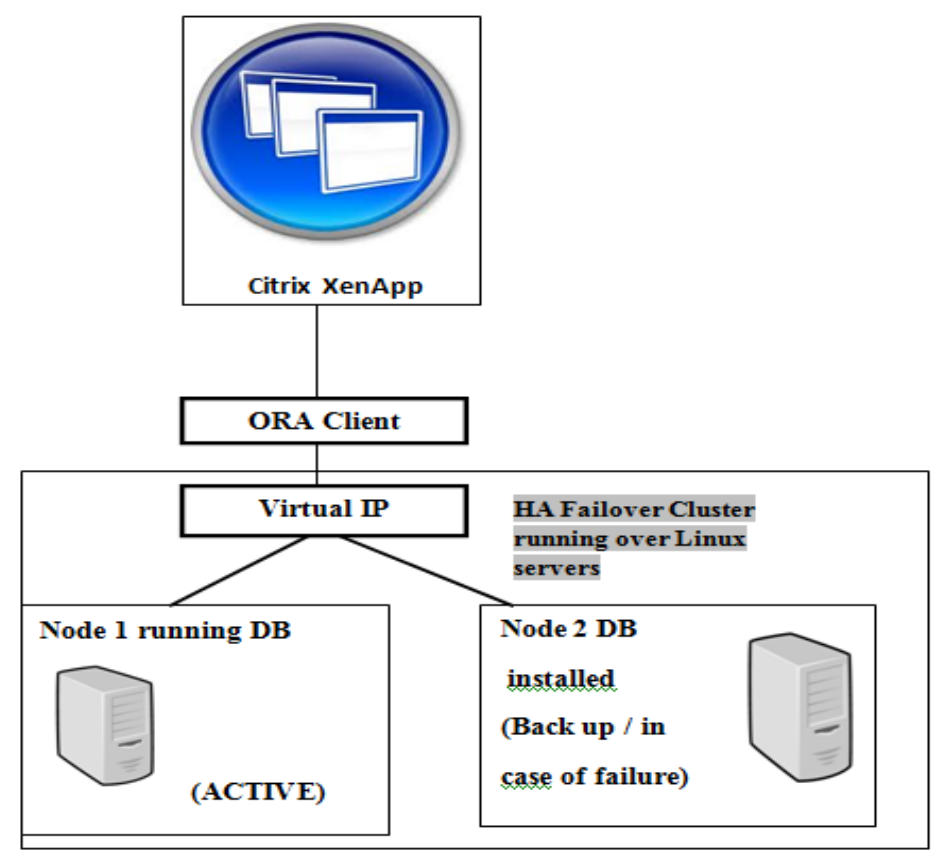

Figure4: Proposed Architecture preventing single point of failure

\section{Conclusion}

For midsize to large enterprises with simple presentation virtualization needs, Terminal Services with XenApp over it fits well. But organizations that must scale to thousands of users will need the compression, acceleration, load balancing, and management options that only XenApp provides has to suffer from single point failure. This new proposed architecture prevents this by introducing an interface and clustering of database servers which dynamically switches at the time of need. Failover clusters provide high-availability support in contrast to database mirroring, which provides high-availability support for a single database. This increases stability and improves performance as the problem of frequently hanging and lack of resource utilization will be solved.

\section{REFERENCES}

[1] “XenApp_on_ESX_Case_Study_June_2010” white paper (Jun. 2010) http://www.vmware.com/files/pdf/techpaper/XenApp_on_ESX_Case_Study_June_2010.pdf

[2] "What's New in VMware vSphere 4: Performance Enhancements" white paper http://www.vmware.com/files/pdf/VMware-vSphere-Performance-WP-EN.pdf

[3] "Design and Scalability Considerations for Enterprise XenApp Deployments" white paper: http://support.citrix.com/servlet/KbServlet/download/28911-102-671619

[4] Wikipedia - Citrix XenApp (Mar. 22, 2013) http://en.wikipedia.org/wiki/Citrix_XenApp

[5] "Citrix XenApp amp XenDesktop" presentation (Mar.12, http://www.slideworld.com/slideshow.aspx/Citrix-XenApp-amp-XenDesktop-ppt-2176421

[6] "Citrix XenApp by Syed" (Dec. 9, 2012) http://www.authorstream.com/Presentation/syedsameer1549058-citrix-XenApp-syed/

[7] "Understanding-the-Citrix-Product-

Portfolio"(Feb.21st,2011) http://vknowledge.wordpress.com/2011/02/21/understanding-the-citrix-product-portfolio/

[8] "How to Choose a Hybrid Cloud Approach" (Second Edition) E-publication(Apr.2012) www.techtarget.com 
International Journal of Information Technology Convergence and Services (IJITCS) Vol.3, No.3, June 2013

[9] “Application virtualization comparison: XenApp vs. ThinApp vs. App-V” (Jan. 2013) http://searchvirtualdesktop.techtarget.com/feature/Application-virtualization-comparison-XenApp vs-ThinApp-vs-App-V

[10] Wikipedia-Independent_Computing_Architecture(May,2010)) http://en.wikipedia.org/wiki/Independent_Computing_Architecture

[11] "XenApp Introduction"Wizz (Apr. 08, 2011) http://vnewbie.com/?page_id=104

[12] “ Product Documentation” Citrix(Dec. 2011) http://support.citrix.com/proddocs/topic/XenApp65w2k8/ps-system-requirements-w2k8-xa65.html

[13] "Citrix - Terminal Server" (Jun. 12, 2012) http://www.tau.ac.il/cc/software/citrix-eng.html

[14] "XenApp 6.0 in the cloud" (Apr 5, 2011) http://blogs.citrix.com/2011/04/05/xenapp-6-0-in-the-cloud/

[15] "XenApp 5 Feature Pack 3 for Windows Server 2003" (Dec. 12, 2012) http://support.citrix.com/proddocs/topic/xenapp/xenapp5fp3-w2k3-wrapper.html

[16] Isha, Chander Kant "An Improved XenApp Architecture" International Journal for advance Research in Engineering and Technology ISSN: 2320-6802" Vol. 1, Issue IV, May 2013, pp 6-9 